\title{
Teoria e prassi: note sulla questione della tragicommedia pastorale in Italia e in Francia
}

Gabriele Niccoli

È generalmente ben risaputo che durante il secondo Cinquecento italiano le teorie dell'arte poetica vengono finalmente studiate ed elaborate in una chiave piú attenta e sistematica. È un periodo, questo, di astiose querelles letterarie e di scottanti dibattiti di critica prammatica. Si formulano, inoltre, nuove regole e nuovi criteri che saranno destinati a tracciare un lungo e, a volte, tortuoso percorso sulla scena della produzione letteraria in Italia ed altrove.

La Poetica aristotelica resta, insieme all'Ars poetica oraziana, il maggior punto di riferimento in questo processo di elaborazione critica. Bisogna per ricordare che non è tanto il trattatello del sommo filosofo ad attizzare le pi disparate controversie letterarie quanto i numerosi commentari ispiratisi all'antico testo e composti durante questo periodo. Molto spesso, infatti, la Poetica viene malintesa o addirittura usata male arbitrariamente allo scopo di poter sostenere particolari teorie letterarie. In quanto all'Ars poetica, la quale godeva già di grande popolarità ancor prima della seconda metà del Cinquecento, essa viene spesso ed esclusivamente adoperata per promuovere gli stessi principî morali propagandati da una Chiesa militante e piú trionfalmente controriformista.

I drammaturghi di quest'epoca rivolgono la loro attenzione in modo particolare allo sviluppo del pensiero critico contemporaneo, soffermandosi inoltre su problemi concernenti l'architettura scenica e su altre preoccupazioni di carattere tecnico e psicologico fra le quali, ad esempio, lo studio (quasi a livello di sondaggio) del pubblico e delle sue aspettative. I teorici ed $\mathrm{i}$ drammaturghi italiani del pieno Cinquecento non tardano a riconoscere che i generi drammatici già ben affermati e provati quali le tragedie e le commedie erudite non riescono piú ad attrarre un gran numero di spettatori e verso la fine del secolo si assiste ad una vera e propria presa di coscienza da QUADERNI d' italianistica Volume VIII, No. 2, 1987 
parte di questi addetti ai lavori al fine di incrementare la popolarità del teatro. Angelo Ingegneri, ad esempio, sostiene che sia la tragicommedia che il dramma pastorale sono non soltanto preferibili, come mezzi teatrali, alle inflessibili e pompose commedie e tragedie erudite ma anche piú idonei ad interessare il pubblico e ad assicurarsi cosí un maggior sostegno finanziario (Weinberg, A History II: 1090; Ingegneri 15-16). È nell'ambiente culturale e letterario appena schematicamente illustrato che fa le sue prime mosse il genere della tragicommedia pastorale. Le origini di questa forma artistica sono state di già delineate da insigni studiosi quali Gregg e Marsan. Piú recentemente, alcuni critici fra i quali Perella, Dalla Valle, Cremona e Lazard hanno dimostrato in qual misura questo nuovo genere drammatico italiano, esemplificato in particolare dall'Aminta e dal Pastor Fido, viene apprezzato e popolarizzato in Francia dove i due capolavori pastorali italiani svolgono un'azione determinante nella creazione di un nuovo tipo di spettacolo che già nei primi decenni del Seicento si sarà confermato insieme alle altre maggiori forme drammatiche. Non sarà nostro compito seguire, quindi, nei suoi dettagli, l'evoluzione della tragicommedia pastorale in Italia e in Francia durante il Cinquecento ed il primo Seicento. Ci limiteremo invece a segnalare alcune componenti di tre tragicommedie pastorali composte durante questo periodo, cercando nel contempo di dimostrare la misura in cui esse rimangono fedeli alle teorie letterarie del tempo. L'argomento è, a nostro avviso, di considerevole interesse non soltanto per ciò che potremmo definire la sua importanza locale nella storia letteraria ma anche, e maggiormente, data la mancanza di una documentazione sistematica in questo campo, per il modello che esso fornisce del singolare rapporto fra teoria e prassi nel tardo Cinquecento.

Giovan Battista Guarini è l'unico drammaturgo che senta il bisogno di dover difendere la definizione del suo Pastor Fido come "tragicommedia pastorale." A questo punto potrebbe sembrare arbitrario categorizzare l'Aminta del Tasso e la Bergerie del Montchréstien (le altre due pastorali sotto analisi) come "tragicommedie pastorali." David Orr, nel suo succinto studio sul dramma rinascimentale italiano in Inghilterra, fa osservare che il dramma pastorale è per necessità una tragicommedia, mentre l'ordine inverso non risulta necessariamente in una analoga illazione (73). ${ }^{1}$ Luigi Fassò sembra 
voler echeggiare una simile opinione allorché, discutendo nel suo Teatro del Seicento del tentativo da parte del Guarini di identificarsi come l'inventore del nuovo genere tragicomico pastorale, afferma che l'autore del Pastor Fido deve assumere questo atteggiamento dal punto di vista polemico ma che, in realtà, egli si muove "in un solco già ben tracciato e fecondato da altri poeti della sua età" (xii).

La "favola boschereccia"-cosí desidera chiamare la sua operetta il Tasso-viene concepita per l'elegante ed aristocratica corte di Ferrara i cui membri la vedono per la prima volta rappresentata sullo sfondo dello stupendo isolotto di Belvedere nel 1573. È da notare che sebbene l'Aminta diventi istantaneamente il prototipo del nuovo genere, questo "portento," come piacque tanto definirla al Carducci, non provoca nessuna controversia o disputa fra i critici contemporanei. Va detto che la critica sull'Aminta non ha, ancor oggi, sottolineato in modo soddisfacente il fatto che l'opera tassesca costituisce un serio tentativo da parte del suo autore di voler formalizzare un nuovo genere drammatico. Ciò è possibilmente dovuto al fatto che nei suoi scritti teorici il Tasso non tratta la questione della tragicommedia pastorale e che i critici suoi contemporanei non hanno creduto opportuno sottoporre la favola ad una analisi completa. Nondimeno, è stato accuratamente osservato che benché Guarini fornisse molti dei principî teorici, fu in realtà la poesia del Tasso a dare vita al dramma pastorale in Italia (Herrick 128). Faremo notare che, al tempo della composizione dell'Aminta, il Tasso non sente il bisogno di fornire alcuna definizione dei principî critici dell'arte drammatica. Il sottotitolo stesso di "favola boschereccia" è alquanto ambiguo. L'Aminta è strutturata nella maniera della tragedia classica.

Il Tasso, in effetti, si fonda sull'autorevole validità della struttura drammatica della tragedia rinascimentale per dar vita ad una creazione artistica nuova e molto apprezzata dai suoi contemporanei. Da un punto di vista puramente esterno, si può osservare che il dramma è preceduto da un prologo, viene sviluppato nello spazio di cinque atti, ciascuno dei quali termina con le parole di un coro (che partecipa, inoltre, all'azione della favola) e si conclude con un epilogo. La regolarità esterna del dramma riflette una serrata struttura interna la quale, fra l'altro, manifesta la doverosa osservanza dell'aristotelica unità dell'azione. Cosciente dello spirito di un altro inestimabile principio aristotelico, il poeta estense adegua il linguaggio della sua 
opera alla particolare natura dell'azione drammatica. Ma non è soltanto l'uso del linguaggio a rinsaldare l'unità strutturale dell'Aminta. Il poeta riesce ad effettuare un felice connubio di elementi puramente fantastici (come, ad esempio, la scena arcadica, l'uso di pastori e di ninfe, il satiro e simili convenzioni pastorali) con degli altri convenevoli alla rigorosa regola della verosimiglianza. Castelveltro, uno dei piú autorevoli critici del tempo, è anche uno dei maggiori sostenitori di questo principio, e il Tasso conosce approfonditamente e rispetta il pensiero dell'illustre critico. Al livello prammatico, le azioni di Silvia e di Aminta sono nella loro essenza piú che probabili. Si può constatare che nell'Aminta la struttura classica della tragedia è utilizzata con successo per dar vita ad una creazione artistica che è essenzialmente rimossa dalla maniera, dallo spirito e dall'espressione che prevalgono nella tragedia. Come abbiamo accennato, i meriti dell'opera tassesca vengono immediatamente riconosciuti dai contemporanei. L'Ingegneri intona la sua categorica approvazione proclamando che l'Aminta è l'opera che finalmente conferma "questa terza specie di dramma, prima o non ricevuta o non apprezzata od almeno non posta nella guisa in uso che s'è fatto da allora in qua" (15).

Il Guarini dà inizio al suo Pastor Fido col preciso intento di voler non solo emulare ma persino sorpassare il suo leggendario contemporaneo. Nella "tragicommedia pastorale" del Guarini (è questo l'attributo specifico sul quale vuole insistere il poeta) la semplice trama tassesca diventa complessa e appesantita; di conseguenza la struttura drammatica comporta una maggiore elaborazione. L'astuto poeta alla Corte estense, muovendosi in un ambiente sentitamente posttridentino e desideroso di legittimare ufficialmente il nuovo genere drammatico, arricchisce, come si è appena fatto notare, la sua opera di complesse trame e di barocchismi verbali che esasperano il buon senso classico dei suoi aristotelici rivali. I dibattiti polemici sul $\mathrm{Pa}$ stor Fido hanno inizio subito dopo la sua stesura, ancora in forma Inanoscritta, nel 1585, si prolungheranno fino ai primi decenni del Seicento e, in effetti, molte di queste dispute avranno un impatto non indifferente sullo sviluppo della critica drammatica francese nei primi decenni di quel secolo. Lo scopo del Guarini è quello di incrementare la popolarità della tragicommedia pastorale utilizzando un'intricata struttura di molteplici azioni amorose (Amarilli-Mirtillo, 
Dorinda-Silvio, Corisca-Mirtillo) insieme ad una gamma di elementi comici molto piú scenicamente efficaci di quelli adoperati dal Tasso. Ma è anche utile sottolineare che, in questo suo procedimento, l'autore del Pastor Fido non si lascia certo trascinare da un eccesso di originalità. A questo proposito Louise George Clubb ha giustamente affermato:

Italian pastoral drama in the mass . . i is essentially a comic genre, as appears not only in the inherent happiness of the pastoral world ... but also in the theoretical articulation of the pastoral attitudes. $(48)^{2}$

La storia della querelle italiana sulla questione della tragicommedia pastorale è stata ampiamente documentata da quasi tutti gli studiosi del Pastor Fido. Allo scopo immediato di questa nostra nota sarà sufficiente ricordare soltanto alcuni degli scambi fra il Guarini e Giason De Nores il quale, da ortodosso aristotelico, non crede affatto nella possibilità di fusione fra tragedia e commedia. Guarini, al contrario, dichiara che l'arte poetica è sottoposta ad uno sviluppo costante, sottolineando cosí l'importanza della prassi nel precedere le regole ed i precetti che codificheranno un particolare genere letterario. Non è forse vero, fa notare argutamente il nostro poeta, che Aristotele stesso ha formulato le sue norme su esempi offertigli dalla prassi della letteratura greca? Il nuovo genere drammatico ha di già una propria identità artistica la cui natura è determinata da un abile adattamento di elementi tragici e comici i quali, aggiunge il nostro poeta, "verisimilmente possano star insieme" (224). ${ }^{3}$ Il De Nores insiste inoltre sulla subordinazione della poesia alla filosofia morale. Secondo questo critico, Aristotele autorizzerebbe soltanto quei generi che propongono una utilità etica o politica. La diatriba fra Guarini e De Nores costituisce, in effetti, un importantissimo capitolo della querelle des Anciens et des Modernes la quale sarà oltremodo significativa nei saloni letterari del Seicento francese. Diversi altri teorici e hommes de théâtre del tardo Rinascimento italiano prendono parte alla disputa mentre i "Moderni" asseriscono vigorosamente che è il gusto del tempo a legittimare la nascita e la costituzione della tragicommedia pastorale. Un patrocinatore dei nuovi orientamenti critici come l'Ingegneri sottolinea un principio di già sostenuto da altri critici: il fatto che il divertimento o il diletto del pubblico sia il fine da raggiungere. Da regista e coreografo, e con una ottima conoscenza delle necessità e considerazioni pratiche della scena, elementi che 
a loro volta saranno anch'essi fondamentali per la formazione del teatro neoclassico francese, l'Ingegneri conclude che la tragicommedia pastorale rimane l'unico mezzo drammatico idoneo a captare e riverberare contemporaneamente mutamenti nel costume politicoculturale, nonché l'ampliamento delle forme letterarie (Weinberg, $A$ History II: 1090-91). Facendo particolare attenzione ai principî di decoro e di verosimiglianza, il Guarini riesce, nel suo Pastor Fido, a fondere felicemente elementi comici e tragici in tal modo da procurare un alto grado di diletto per il suo pubblico. L'insistenza che il poeta pone, infatti, su questo aspetto non è che uno dei piú cocenti preludi all'estetica barocca che si va materializzando in questo periodo. Il nostro drammaturgo è meticolosissimo nella elaborazione del suo linguaggio drammatico e, col preciso intento di far divertire il pubblico, egli armonizza il linguaggio con lo spirito del mondo pastorale. L'accurata scelta di questo particolare linguaggio, allo stesso tempo, provoca nell'opera del Guarini un eccesso di giochi retorici, di astute metafore, di pointes e di concettismi. ${ }^{4}$

L'enorme ed immediata popolarità riscossa dai due capolavori pastorali italiani in Francia è ben nota. In un ancor recente studio sulla Bergerie di Antoine de Montchréstien, Isida Cremona è dell'avviso che questo dramma, composto fra il 1597 e la fine del secolo, segni il punto di transizione fra le prime imitazioni francesi e le pastorali dell'epoca seguente le quali si ispirano a fonti piú disparate $(50){ }^{5}$ La pastorale rappresenta il maggiore sviluppo della letteratura drammatica nella nuova Francia di Henri IV; essa costituisce, inoltre, il mezzo piú adeguato alla sperimentazione teatrale. ${ }^{6}$ Epperò un problema ancora da risolvere in modo esauriente nella storia letteraria del tardo Cinquecento francese riguarda la misura in cui i drammaturghi di quel periodo, e piú precisamente degli ultimi due decenni del sedicesimo secolo, osservino principî critici elaborati in Francia. I teorici francesi dell'ultimo Cinquecento si occupano maggiormente e del linguaggio, il quale è ancora in fase di sviluppo, e del verso le cui regole e forme saranno poi legittimate da Malherbe. A chi si rivolgono drammaturghi quali il Montchréstien per trovare le formule teoriche da applicare alla stesura dei loro drammi pastorali? René Bray fornisce un'accurata risposta, anche se misera nei dettagli, quando afferma che nel tardo Cinquecento francese ". . . les maîtres de poétique que l'on cherche et que l'on ne trouve pas en France, on les 
demande et on les trouve à l'étranger" (24). ${ }^{7}$ Occupandosi essenzialmente della questione della lingua e seguendo le orme del Du Bellay e del Ronsard in quanto all'ancor vivo esempio della "lustration,"alcuni degli scritti teorici che vengono però alla luce poco prima della composizione della Bergerie offrono vari criteri pratici da applicare a particolari generi letterari. Ciò si evidenzia con Pierre Delaudun il quale, nella sua Art poétique française del 1597, esorta il drammaturgo all'imitazione degli aspetti piú riusciti del prototipo di un dato genere. Altri consigli pratici abbondano e, benché non si menzionino fini utilitari, il teorico francese afferma che il fine della poesia è il diletto del pubblico (Weinberg, Critical Prefaces 38-41). ${ }^{8}$ Un'altra Art poétique française, di Vauquelin de la Fresnaye, pubblicata nel 1605 ma composta diversi anni prima della fine del secolo, è essenzialmente un'elaborazione dell'opera oraziana, ma in essa Vauquelin insiste sulla necessità di adeguare il linguaggio alla natura del genere-si adopera il termine "convenant" - sul principio dell" utile dulci. Vauquelin si dichiara convinto che un dramma deve essere sviluppato non secondo principî di struttura interna ma secondo un certo numero di convenzioni e di precedenti storici in quel particolare genere drammatico (Weinberg, Critical Prefaces 51). Non crediamo sia del tutto inutile soffermarsi, come sembra opinare la critica letteraria sul Montchréstien, sulla familiarità o meno che il misterioso tragediografo e avventuriero francese abbia potuto avere con tali precetti teorici $o$, in una opzione favorevole, quale influenza essi abbiano potuto esercitare sulla composizione della sua Bergerie. Una attenta ed approfondita lettura dell'opera pastorale francese ci fa comunque intravvedere la maniera in cui il Montchréstien riesce a mettere in pratica i pochi suggerimenti appena accennati. Il drammaturgo francese non esita minimamente, ad esempio, a fare ampio uso dei migliori modelli di tragicommedia pastorale nel restare fedele agli schemi stilistici e strutturali dell'Aminta e del Pastor Fido. Egli adopera un linguaggio interamente adeguato, o "convenant," al genere pastorale drammatico e mette in atto una vasta gamma di convenzioni ed elementi tradizionali del mondo arcadico. ${ }^{9}$ Il vago e generico titolo dell'opera sembra tradire una sorta di insicurezza da parte del drammaturgo nella identificazione di un genere per il quale manca ancora una precisa base teorica in Francia. D'altronde, le teorie del Guarini, nonché i vari principî sostenuti nel corso delle 
dispute italiane sulla tragicommedia pastorale, non sembrano aver influenzato in modo significativo $\mathrm{i}$ teorici e $\mathrm{i}$ drammaturghi francesi fino ai primi decenni del Seicento. La mancanza in Francia di una autorevole teoria drammatica, sistematicamente studiata ed elaborata, è la causa primaria delle ovvie deficienze di carattere strutturale all'interno della Bergerie. Inevitabilmente, la struttura interna del dramma viene indebolita in modo considerevole anche dal fatto che Montchréstien sembra ossessionato (come se dovesse seguire tutti i precetti del Vauquelin ad litteram) a sviluppare la trama basandosi su una vera e propria sequenza di memorabili convenzioni e topoi pastorali. Va peraltro osservato che le maggiori convenzioni ed i maggiori luoghi comuni del mondo arcadico italiano e, in modo particolare, di quello ferrarese come, ad esempio, Amore in abito pastorale, il Satiro, e la "bell'età dell'oro" vengono trasportati sulla scena francese quasi esclusivamente per dar concretezza a precise esigenze di movimento e di vivacità rappresentative. In altre parole, mentre sulla scena italiana tali convenzioni pastorali comportano una determinata e determinante funzione in rapporto allo svolgimento della trama, in Francia si assiste spesso ad una elaborazione autonoma di esse, ad un loro disimpegno dalla struttura interna dell'opera. È ciò che accade, in larga misura, anche nella Bergerie del Montchréstien, la quale, per la verità, deve sostenere non una ma ben quattro trame (una serie di egloghe drammatiche scenicamente autonome). Nei protagonisti del dramma (Fortunian-Dorine), se cosí ci è lecito definirli, dato che essi interessano e si muovono esclusivamente nello spazio scenico-drammatico della loro particolare egloga, si rispecchia tuttavia un lieto affiatamento fra linguaggio lirico di stampo tassesco (Aminta-Silvia) ed elaborazione drammatica modellata sul Guarini (Mirtillo-Amarilli). Montchréstien, motivato dal nuovo ottimismo politico francese alla fine del secolo, ripropone le strutture e gli schemi pastorali dei due geniali scrittori italiani. Ma al di là di qualche felice fusione fra linguaggio altamente lirico e psicologia drammatica, l'opera francese si esprime soltanto nei limiti del ritmo scenico e nella riproduzione prettamente teatrale di topoi e microstrutture, non essendo essa sostenuta dalle fonti critiche che hanno invece caratterizzato la produzione drammatica del tardo Cinquecento italiano.

University of St. Jerome's College

University of Waterloo 


\section{NOTE}

1 Il critico americano constata inoltre che un dramma il quale ". . . is highly romantic in nature, carrying throughout an unrelieved air of tragedy, only to end happily, is a tragicomedy" (96).

$2 \mathrm{La}$ Clubb insiste, giustamente, sugli svariati esperimenti post-tassiani che non poterono non incidere sulla elaborazione del Pastor Fido.

3 Aggiunge il nostro teorico: “. . . perciocché dall'una prende le persone grandi e non l'azione; la favola verisimile, ma non vera; gli affetti mossi, ma rintuzzati; il diletto, non la mestizia; il pericolo, non la morte; dall'altra il riso non dissoluto, le piacevolezze modeste, il nodo finto, il rivolgimento felice, e sopratutto l'ordine comico. ... Le quali parti, in questa guisa corrette, possono stare insieme in una favola sola, quand'elle massimamente sono condite col lor decoro e con le qualitàa del costume che lor convengono" (231).

4 Si tratta in effetti dello "stile mediocre" e, come ci fa osservare il Perella, Lingua e struttura 23, "la natura lirica di questo linguaggio non è estranea al contenuto del Pastor Fido." Sull'claborazione dello stile e del linguaggio nell'opera guariniana, si veda anche Battaglin 291-353.

5 "Pourtant-afferma inoltre la Cremona - par ses thèmes, son esprit et son langage, la Bergerie est encore essentiellement tributaire de l'Aminta." Herrick 143, sostiene che in seguito al successo francese delle due opere italiane, “. . . the simple, incoherent plots of earlier French pastoral drama became better organized, more complex, and the French pastoral drama acquired the characteristics of tragicomedy."

6 Sui dati statistici della sperimentazione teatrale del nuovo genere drammatico pastorale e significativo lo studio di Dabney 449.

7 Aggiunge il noto critico Lancaster I: 27: "there is little evidence to show that writers of the period reflected deeply upon their art ..." sottolineando che molti scrittori si rifanno o ad altri drammaturghi francesi o, molto piú probabilmente, agli autori italiani in quel particolare genere.

8 Cfr. Weinberg Critical Prefaces 38-41. Lo stesso Delaudun, nella composizione della sua bergerie, ha voluto procedere, ci confessa, imitando “. . . un docte et gentil esprit." $\mathrm{Ci}$ viene, fra l'altro, anche fatto notare che è bene che la bergerie contenga "un sens moral."

9 Altemandosi felicemente fra il verso e la prosa, Marsan 221, sottolinea la natura lirica di questa stupenda prosa. Montchréstien, ancora una volta nelle parole del critico francese "prend son bien où il le trouve et il le trouve partout" (233). E subito aggiunge: "Les personnages semblent avoir vécu dans l'intimité des héros de Guarini, du Tasse. ..." 


\section{OPERE CONSULTATE}

BATTAGLIN, D. "Il linguaggio tragicomico del Guarini e l'elaborazione del Pastor Fido." Lingua e strutture del teatro italiano del Rinascimento. Padova: Liviana, 1970.

BRAY, R. La formation de la doctrine classique en France. Paris: Nizet, 1951.

CLUBB, G. L. "The Making of the Pastoral Play: Some Italian Experiments between 1573 and 1590." Italian Criticism and Theatre: from Petrarch to Pirandello. Ed. J. A. Molinaro. Toronto: U of Toronto P, 1973.

CREMONA, I. L'influence de l" "Aminta" sur la pastorale dramatique française. Paris: Vrin, 1977.

DABNEY, L. E. French Dramatic Literature in the Reign of Henri IV. Austin: University Cooperative Society, 1952.

DALla VALLE, D. Pastorale barocca. Forme e contenuti dal "Pastor Fido" al dramma pastorale francese. Ravenna: Longo, 1973.

FASSÒ, L. Teatro del Seicento. Milano: Ricciardi, 1956.

GREGG, W. W. Pastoral Poetry and Pastoral Drama. New York: Russel \& Russel, 1959.

GUARINI, G. Il Pastor Fido e il Compendio della poesia tragicomica. Ed. G. Brognolino. Bari: Laterza, 1914.

HERRICK, M. T. Tragicomedy, its Origin and Development in Italy, France, and England. Urbana: U of Illinois P, 1955.

INGEGNERI, A. "Della poesia rappresentativa e del modo di rappresentare le favole sceniche." Lo spettacolo dall' umanesimo al manierismo. Ed. F. Marotti, Milano: Feltrinelli, 1974: 15-16.

LANCASTER, H. C. A History of French Dramatic Literature in the Seventeenth Century. Vo1. 1. Baltimore: The Johns Hopkins U P, 1929.

LAZARD, M. Le théâtre en France au XVIème siècle Paris: Presses Universitaires de France, 1980.

MARSAN, J. La pastorale dramatique en France d la fin du XVleme et au commencement du XVIIIeme siecle. Paris: Hachette, 1905.

ORR, D. Italian Renaissance in England before 1625. Chapel Hill: U of North Carolina P, 1970.

PERELLA, N. J. The Critical Fortune of Battista Guarini's "Il Pastor Fido." Firenze: Olschki, 1973.

WEINBERG, B. Critical Prefaces of the French Renaissance. Evanston: Northwestern U P, 1950.

A History of Literary Criticism in the Italian Renaissance. 2 vols. Chicago: U of Chicago P, 1961. 